\title{
Primary mucinous adenocarcinoma of the left renal pelvis with ectopic inferior vena cava and invasion of the left renal vein and the adjacent inferior vena cava: a case report
}

\author{
Bo Han ${ }^{1}$, Qian Xie ${ }^{2}$, Maozhi Tang ${ }^{1}$, Hongwen Zhao ${ }^{1}$, Xiaosong $\mathrm{Xu}^{1}$ \\ ${ }^{1}$ Department of Nephrology, First Affiliated Hospital (Southwest Hospital), Army Medical University (Third Military Medical University), \\ Chongqing, China; ${ }^{2}$ Neonatal Department, Chongqing Health Center for Women and Children, Chongqing, China \\ Correspondence to: Xiaosong Xu, MD. Department of Nephrology, First Affiliated Hospital (Southwest Hospital), Army Medical University (Third \\ Military Medical University), Gaotan Yan Street No. 36, Shaping Ba District, Chongqing 400038, China. Email: xxs201108@163.com.
}

\begin{abstract}
Primary mucinous adenocarcinoma of renal pelvis is an extremely uncommon malignant tumor without typical clinical manifestations and imaging characteristics. A definite diagnosis often depends on postoperative pathological results. Operation is the preferred choice of treatment, but prognosis is unsatisfactory. We describe a 42-year-old male patient who was admitted for repeated and intermittent pain of left abdominal flank for more than 5 years and aggravation of the symptom for more than 1 month. In the course of disease, he was misdiagnosed twice as a renal cyst in other hospitals. However, mild percussive pain was discovered in the left kidney area during this hospitalization. Moreover, abdominal computed tomography (CT) scan of our hospital demonstrated that a huge mixed-density mass derived from left kidney, along with congenital variation of the inferior vena cava and filling defect area in the left renal vein and the adjacent inferior vena cava. After adequate preoperative preparation, he was treated with radical resection of the left kidney and artificial vascular replacement of the inferior vena cava segment containing the emboli. The mass was verified to be mucinous adenocarcinoma by postoperative pathological result. In the end, he was diagnosed as primary mucinous adenocarcinoma of the left renal pelvis with ectopic inferior vena cava and invasion of the left renal vein and the adjacent inferior vena cava. Two weeks after operation, he recovered and was discharged. There was no evidence of recurrence after more than 4 years of followup. Blood oncogenic biomarkers were valuable in diagnosis by reviewing literature. In conclusion, Primary mucinous adenocarcinoma of the kidney is easy to be misdiagnosed as renal cyst. Preoperative CT and blood oncogenic biomarkers are extremely important for preliminary diagnosis. Postoperative pathological result is the gold standard for final diagnosis. Although prognosis is generally unfavourable, radical resection of the tumor can benefit patients.
\end{abstract}

Keywords: Mucinous adenocarcinoma; renal pelvis; congenital variation; case report; pathological diagnosis

Submitted Apr 25, 2021. Accepted for publication Jul 05, 2021.

doi: $10.21037 /$ tcr-21-719

View this article at: https://dx.doi.org/10.21037/tcr-21-719

\section{Introduction}

Primary mucinous adenocarcinoma of the kidney, lacking typical clinical manifestations and imaging features, is an especially rare malignancy and usually occurs in the renal pelvis $(1,2)$. On the one hand, the patients may be easy to be missed due to having no symptoms in the early stage. On the other hand, ultrasound examinations often show cystic masses with dark areas of anechoic liquid, which are easily misdiagnosed as renal cysts. Before operation, it is particularly difficult to obtain a definite diagnosis in clinical practice (1). The vast majority of patients are diagnosed according to pathological results of specimens after surgical treatment $(2,3)$. Up to now, there is a lack of other secure and effective therapy methods except 
operation (4). Moreover, prognosis of the patients is generally unfavourable, with about $50 \%$ of patients dying within 2 years after operation $(5,6)$. Therefore, it is still of great practical value to report new case and review the previous literatures in order to provide more clinical experience for management of the disease. Here is a special case of primary mucinous adenocarcinoma of the left renal pelvis, invading the left renal vein and inferior vena cava. The patient exhibited an uncommon congenital variation of the inferior vena cava. Below the level of the right renal vein, its anatomical location was on the left side of the spine, adjacent to the abdominal aorta.

We present the following article in accordance with the CARE reporting checklist (available at https://dx.doi. org/10.21037/tcr-21-719).

\section{Case presentation}

\section{Patient and treatment}

This 42-year-old male patient complained of repeated and intermittent pain of left abdominal flank for more than 5 years and aggravation of the symptom for more than 1 month. At the beginning of 2012, he presented left abdominal flank pain without clear inducements. He had no fever, radiating pain in other areas, irritation sign of bladder, gross hematuria, etc. Ultrasound examination at the local hospital indicated that the upper pole of the left kidney had a cystic anechoic area. It was thought to be a cyst. No treatment was given. Observation and follow-up were recommended. Subsequently, he occasionally manifested left abdominal flank pain. In August 2015, he returned to the local hospital and underwent a needle biopsy of left renal mass under the guidance of ultrasound. The pathological result showed that the mass contained eosinophilic mucus with no definite structure. It was again considered as a cyst and surgical treatment was not performed. In March 2017, the patient's symptom worsened after excessive activity and showed no significant relief after enough rest. For definite diagnosis and active treatment, he was admitted to the First Affiliated Hospital of the Army Medical University in April 2017. The vital signs were normal at the time of admission. The cardiopulmonary and abdominal physical examination exhibited no obvious abnormalities. There was no percussive pain in the right kidney area, but mild percussive pain was discovered in the left kidney area. No abnormality was found in blood routine, electrolyte, blood clotting index, liver function, and kidney function. Abdominal computed tomography (CT) scan demonstrated that the mass of the left kidney appeared as mixed density, mainly cystic density mixing with spot-like dense shadows, presenting polycystic changes with thick wall and no obvious enhancement (Figure 1A-1D). Below the level of the right renal vein, the anatomical location of the inferior vena cava was on the left side of the spine. There was filling defect area in the left renal vein and the adjacent inferior vena cava (Figure $1 E, 1 F$ ). Emission computed tomography (ECT) prompted that the blood supply and glomerular filtration function of both kidneys were normal, the right upper urinary tract drainage was unobstructed, and the left upper urinary tract drainage was slightly slow.

Combined with clinical manifestation, physical examination, laboratory tests and imaging changes, a preliminary diagnosis of the patient was considered to be left renal cystic tumor with emboli of left renal vein and inferior vena cava. He was advised to stay in bed to avoid serious cardiovascular complications, such as pulmonary embolism caused by abscission of emboli after excessive activity, and given active anticoagulant therapy. After adequate preoperative preparation, he was treated with radical resection of the left kidney and artificial vascular replacement of the inferior vena cava segment containing the emboli under general anesthesia. Dark red emboli were observed in the excisional inferior vena cava and left renal vein after operation. Anticoagulant therapy was continued to prevent thrombosis after artificial vascular replacement. Two weeks after surgery, he recovered and was discharged. Although prognosis of the patients is generally poor, there was no evidence of recurrence after more than 4 years of follow-up.

\section{Pathology}

On gross examination, the left kidney approximately measured $12 \mathrm{~cm} \times 6 \mathrm{~cm} \times 4 \mathrm{~cm}$. The huge mass was found in the upper pole of the left kidney. After dissection, it was proved that the mass originated from the renal pelvis. Fibrous cords were seen in the mass to divide into cysts of varying sizes, which were filled with gray viscous jelly-like liquid (Figure 2A,2B). Microscopically, the tumor cells were oval or columnar, the cytoplasm was abundant with light staining, and the nuclei were round or polygonal with deep staining. The tumor cells were arranged in irregular glandular ducts or cords, with pools of mucus (Figure $3 A, 3 B$ ). Immunohistochemical experiments revealed that the tumor cells were positive for cytokeratin (CK) (Figure 3C), caudal type homeobox 

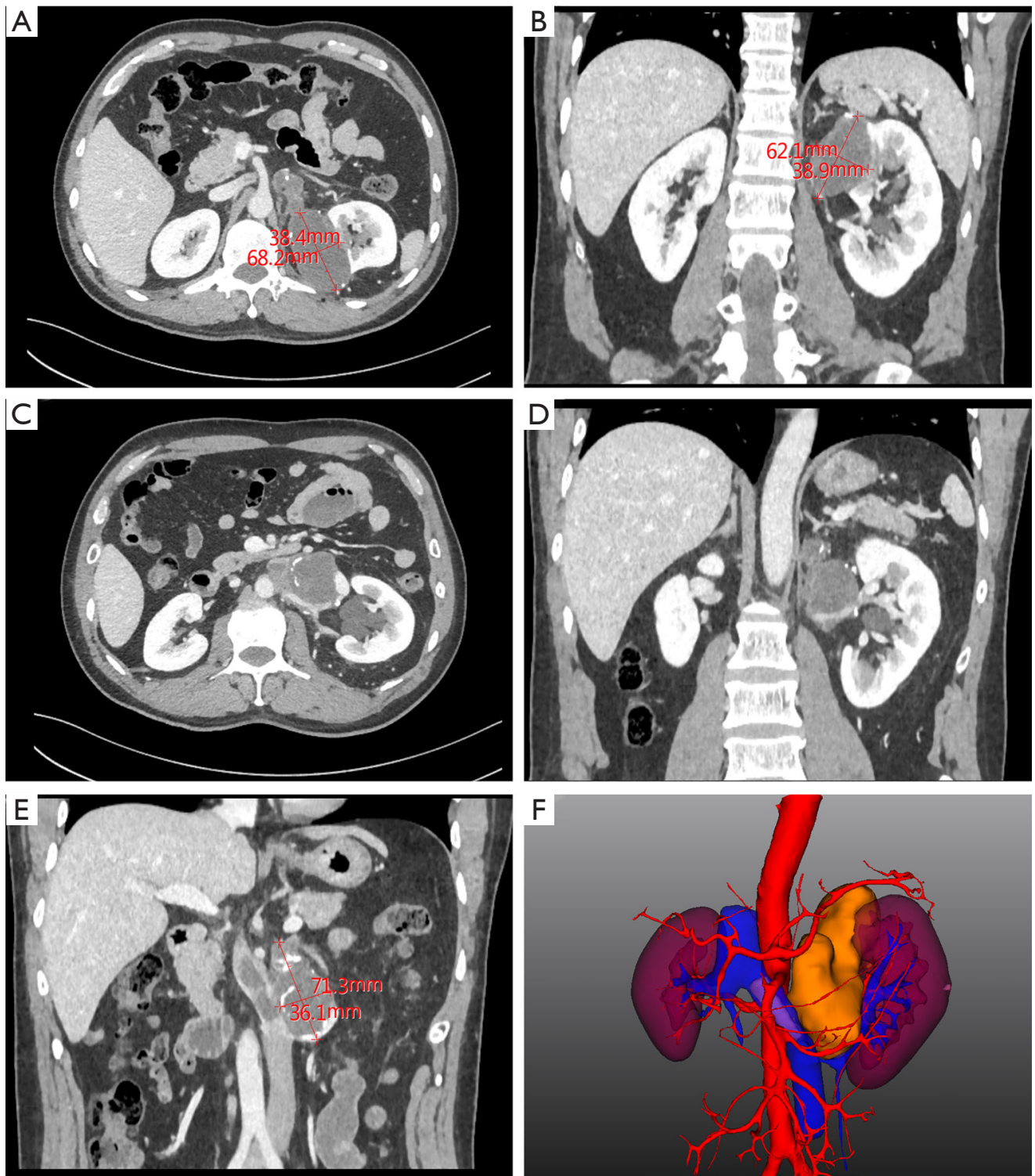

Figure 1 Computed tomography (CT) scan of renal medulla phase of artery angiography. (A,B) The mass was located in the upper pole of the left kidney. (C,D) The blood vessels of the left kidney were severely compressed by the huge mass. (E) The inferior vena cava was ectopic to the left, and its internal filling defect indicated emboli. (F) 3D visualization imaging of the left renal mass and the vessels. The mass was represented by orange tissue. The filling defect area was represented by purple tissue. The anatomic variation of the inferior vena cava was clearly shown again.

2 (CDX-2) (Figure 3D) and carcinoembryonic antigen (CEA), but negative for CK7, CK20, synaptophysin (Syn), chromogranin A (Cg A) and vimentin (VIM). The number of Ki67 positive cells was about $2 \%$. The above atypia cells were also found in the venous emboli. The patient had no history of gastrointestinal disease. On the basis of the histomorphological characteristics and immunohistochemical results, a final diagnosis of primary mucinous adenocarcinoma of the left renal pelvis with invasion of the left renal vein and the inferior vena cava was established.

All procedures performed in studies involving human participants were in accordance with the ethical standards of the institutional research committee(s) and with the 

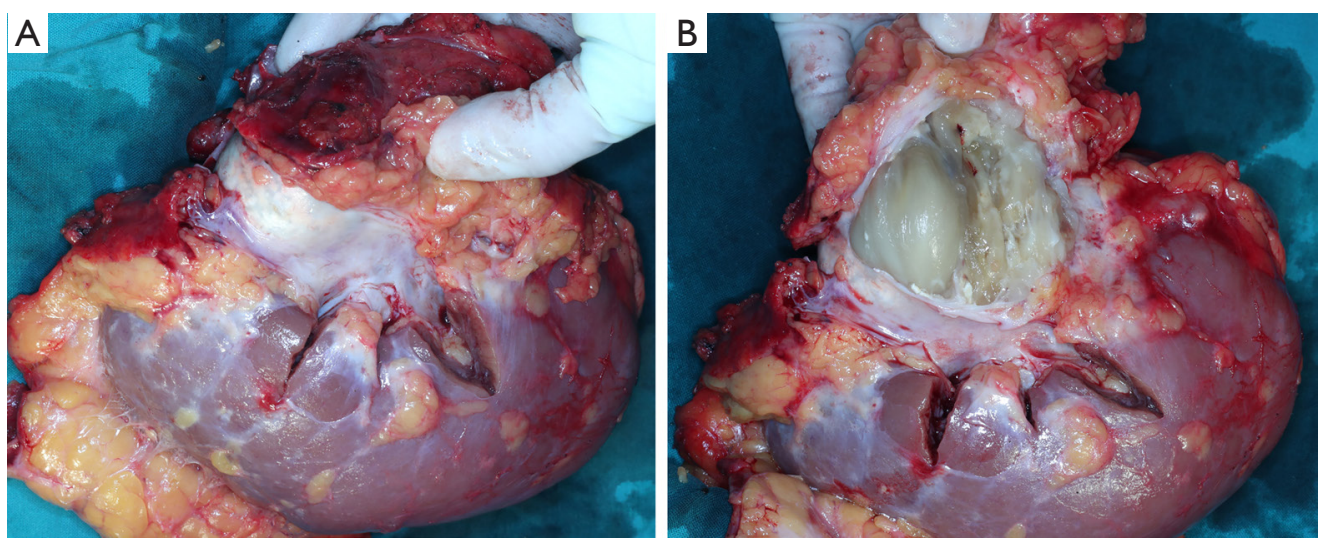

Figure 2 Postoperative images of the left kidney and the mass. (A,B) Histologically, the mass was derived from the renal pelvis.
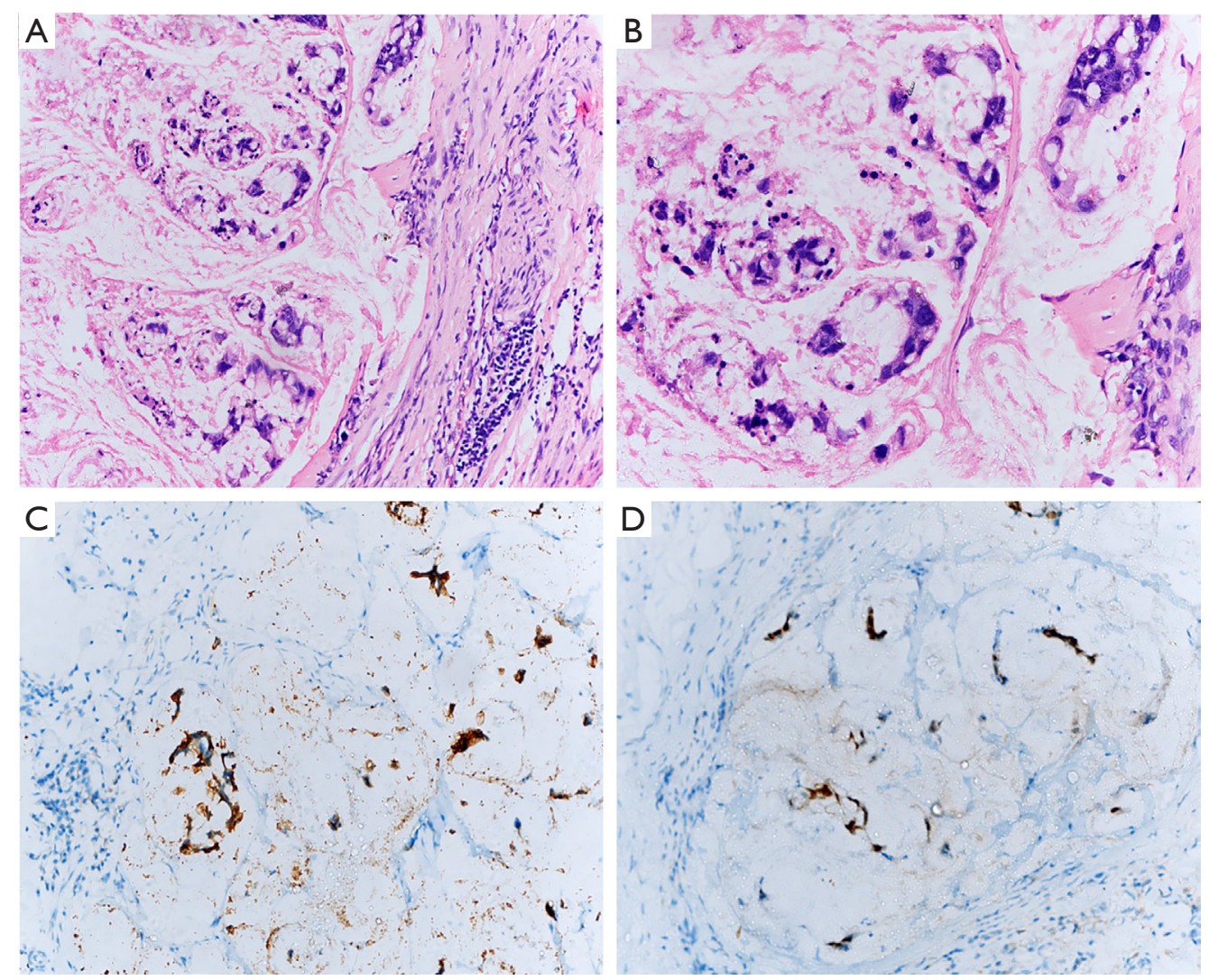

Figure 3 Histochemical staining of the resected mass. (A) Hematoxylin-eosin staining. The arrangement of tumor cells was either glandular or scattered individually $(\times 200)$. (B) Hematoxylin-eosin staining. The tumor cells had large, deeply stained, and irregular nuclei, with less cytoplasm and high nucleocytoplasmic ratio $(\times 400)$. (C) Immunohistochemical staining. The CK staining of tumor cell cytoplasm was positive $(\times 200)$. (D) Immunohistochemical staining. The CDX-2 staining of the tumor cell nuclei was positive $(\times 200)$. 
Helsinki Declaration (as revised in 2013). Written informed consent was obtained from the patient.

\section{Discussion}

Malignant tumors in the renal pelvis mainly originate from epithelial cells of urinary tract, including transitional cell carcinoma, squamous cell carcinoma and adenocarcinoma. Adenocarcinoma of the renal pelvis is the least common type, accounting for less than $1 \%$. It can be further classified as tubulovillous, mucinous, or papillary nonintestinal categories, of which papillary nonintestinal category is the rarest subtype, followed by mucinous category $(2,4,7)$.

In 1960, primary mucinous adenocarcinoma of the renal pelvis was first described by Hasebe et al. (8). To date, it remains an extremely rare tumor with fewer than 100 cases reported (9). At present, the pathogenesis of primary mucinous adenocarcinoma of the renal pelvis is still not clear. However, there are two leading theories widely accepted by most researchers. One theory holds that mucosal epithelium of renal pelvic may undergo glandular metaplasia under long-term chronic irritations such as nephrolithiasis, hydronephrosis and infection, and then abnormal hyperplasia and adenocarcinoma possibly occur $(10,11)$. Because of the increased incidence of the lesions in anomalous kidneys such as horseshoe kidney and non-functioning kidney, the other theory suggests that primary mucinous adenocarcinoma also may arise from the sequestered renal epithelium within the parenchyma as a consequence of maldevelopment (4).

Primary mucinous adenocarcinoma of the renal pelvis lacks typical clinical manifestations. A recent article summarized the conditions of 30 patients reported in literatures since 2000 (1). Eleven cases presented single symptom of abdominal flank pain or discomfort, 3 cases presented single symptom of hematuria, and 2 cases presented single symptom of abdominal mass. Six cases with two or more symptoms mentioned above, only 1 case presented three symptoms, similar to renal cell carcinoma triad, which should be differentiated. The case we reported presented only abdominal flank pain, with no other symptoms. No diagnosis can be made solely on the basis of clinical presentation. In addition, abdominal ultrasonography, intravenous pyelography, abdominal $\mathrm{CT}$ and other imaging examinations may not be able to accurately identify the tumor (12). Radiological findings of previously reported cases revealed that most of the patients presented with hydronephrosis, pyonephrosis and calculi, some patients presented with a mass or tumor, and a few patients presented with obstruction or stenosis of pelvis ureteric junction (1). The patient we reported presented with a huge mass, which was initially misdiagnosed as a renal cyst. The needle biopsy did not show any findings. The possibility of tumor was not suspected until CT examination showed renal mixed density mass accompanied by emboli of left renal vein and inferior vena cava. It is difficult to make accurate diagnosis by preoperative clinical manifestations and imaging examinations. Apparently, postoperative pathological support is indispensably needed. However, a few literatures suggested that primary mucinous adenocarcinoma of the renal pelvis may be accompanied by increased carbohydrate antigen 19-9 (CA19-9) or CEA $(4,13)$. Moreover, combination of imaging examinations and blood oncogenic biomarkers can improve the diagnostic accuracy to some extent (1). Unfortunately, the patient in this study was not examined for biomarkers, and corresponding evidence could not be obtained.

Mucinous adenocarcinoma usually occurs in the colorectal and ovarian regions and is characterized by a large amount of mucinous secretion, accounting for more than $50 \%$ of the tumor volume $(14,15)$. For mucinous adenocarcinoma of the renal pelvis, metastasis of adenocarcinoma in other organs should be given priority. The diagnosis of primary mucinous adenocarcinoma of the renal pelvis can be established only if other organs are excluded (13).

Once the mass is misdiagnosed as a simple cyst or a benign tumor, it may cause treatment delay and serious consequences. Early surgical treatment can not only relieve the symptoms, but also prolong the patient's life span and improve the quality of life (4). Because the overflow of cancer cells during operation or the implantation metastasis along the ureter might cause local recurrence, radical resection was recommended in clinical practice for patients suspected or initially diagnosed with mucinous adenocarcinoma of the renal pelvis (2,5-7). The kidney, ureter and bladder cuff on the affected side required radical resection (16). Previously, researchers had made some attempts on adjuvant therapy after operation, such as interleukin-2, radiotherapy and capecitabine $(4,6,17)$. However, up to now, there is no conclusive evidence of the effectiveness of those methods, which needs further research and exploration.

In conclusion, primary mucinous adenocarcinoma of the renal pelvis is a rare and malignant tumor. It is pretty difficult to be diagnosed preoperatively. 
However, some clues may be found through integrating imaging examinations and blood oncogenic biomarkers. Postoperative pathological result is the gold standard for final diagnosis. Operation is currently the preferred treatment method and radical resection of the tumor can benefit patients.

\section{Acknowledgments}

Funding: None.

\section{Footnote}

Reporting Checklist: The authors have completed the CARE reporting checklist. Available at https://dx.doi. org/10.21037/tcr-21-719

Conflicts of Interest: All authors have completed the ICMJE uniform disclosure form (available at https://dx.doi. org/10.21037/tcr-21-719). The authors have no conflicts of interest to declare.

Ethical Statement: The authors are accountable for all aspects of the work in ensuring that questions related to the accuracy or integrity of any part of the work are appropriately investigated and resolved. All procedures performed in studies involving human participants were in accordance with the ethical standards of the institutional and/or national research committee(s) and with the Helsinki Declaration (as revised in 2013). Written informed consent was obtained from the patient for publication of this case report and accompanying images. A copy of the written consent is available for review by the editorial office of this journal.

Open Access Statement: This is an Open Access article distributed in accordance with the Creative Commons Attribution-NonCommercial-NoDerivs 4.0 International License (CC BY-NC-ND 4.0), which permits the noncommercial replication and distribution of the article with the strict proviso that no changes or edits are made and the original work is properly cited (including links to both the formal publication through the relevant DOI and the license). See: https://creativecommons.org/licenses/by-nc-nd/4.0/.

\section{References}

1. Li H, Xie F, Zhao C, et al. Primary mucinous adenocarcinoma of the renal pelvis misdiagnosed as calculous pyonephrosis: a case report and literature review. Transl Androl Urol 2020;9:781-8.

2. Han DS, Yuk SM, Youn CS, et al. Primary mucinous cystadenocarcinoma of the renal pelvis misdiagnosed as ureteropelvic junction stenosis with renal pelvis stone: a case report and literature review. World J Surg Oncol 2015;13:324.

3. Lee HY, Jang MY, Wu WJ, et al. Primary mucinous adenocarcinoma of the renal pelvis. Urol Sci 2014;25:65-7.

4. Lai C, Teng XD. Primary enteric-type mucinous adenocarcinoma of the renal pelvis masquerading as cystic renal cell carcinoma: A case report and review of the literature. Pathol Res Pract 2016;212:842-8.

5. Sisodia SM, Khan WA, Bhavsar SP. Incidental primary papillary mucinous adenocarcinoma of the renal pelvis in a case of non-functioning kidney due to chronic pyelonephritis and pelvic calculus. Saudi J Kidney Dis Transpl 2012;23:592-3.

6. Raphael V, Sailo S, Bhuyan A, et al. Mucinous adenocarcinoma of the renal pelvis with adenocarcinoma in situ of the ureter. Urol Ann 2011;3:164-6.

7. Abbas M, Kramer MW, Spieker T, et al. Primary mucinous adenocarcinoma of the renal pelvis with carcinoma in situ in the ureter. J Egypt Natl Canc Inst 2014;26:51-4.

8. Hasebe $M$, Serizawa $S$, Chino S. On a case of papillary cystadenocarcinoma following malignant degeneration of a papillary adenoma in the kidney pelvis. Yokohama Med Bull 1960;11:491-500.

9. Shah VB, Amonkar GP, Deshpande JR, et al. Mucinous adenocarcinoma of the renal pelvis with pseudomyxoma peritonei. Indian J Pathol Microbiol 2008;51:536-7.

10. Rao P, Pinheiro N Jr, Franco M, et al. Pseudomyxoma peritonei associated with primary mucinous borderline tumor of the renal pelvicalyceal system. Arch Pathol Lab Med 2009;133:1472-6.

11. Patel KN, Patel NA, Gandhi SP. Presentation of mucinous adenocarcinoma of renal pelvis masquerading as gross hydronephrosis: A histopathological surprise. South Asian J Cancer 2017;6:78.

12. Kaur G, Naik VR, Rahman MN. Mucinous adenocarcinoma of the renal pelvis associated with lithiasis 
and chronic gout. Singapore Med J 2004;45:125-6.

13. Ho CH, Lin WC, Pu YS, et al. Primary mucinous adenocarcinoma of renal pelvis with carcinoembryonic antigen production. Urology 2008;71:984.e7-8.

14. Hugen N, Brown G, Glynne-Jones R, et al. Advances in the care of patients with mucinous colorectal cancer. Nat Rev Clin Oncol 2016;13:361-9.

15. Morice P, Gouy S, Leary A. Mucinous Ovarian Carcinoma. N Engl J Med 2019;380:1256-66.

Cite this article as: Han B, Xie Q, Tang M, Zhao H, Xu X. Primary mucinous adenocarcinoma of the left renal pelvis with ectopic inferior vena cava and invasion of the left renal vein and the adjacent inferior vena cava: a case report. Transl Cancer Res 2021;10(9):4243-4249. doi: 10.21037/tcr-21-719
16. Takehara K, Nomata K, Eguchi J, et al. Mucinous adenocarcinoma of the renal pelvis associated with transitional cell carcinoma in the renal pelvis and the bladder. Int J Urol 2004;11:1016-8.

17. Higgins A, Eisa W, Walton J, et al. Metastatic Mucinous Adenocarcinoma and Carcinoid Tumor Arising From a Mature Cystic Teratoma of a Horseshoe Kidney. Urol Case Rep 2017;11:39-41. 